\title{
Chemical Cross-Linked Gelation of Hydroxypropyl Guar (HPG) under Shear Flow
}

\author{
Imshik LeE, Chunli BAI, Xinwen WANG, and Nai-Xin WANG \\ STM Laboratory, Institute of Chemistry, Chinese Academy of Sciences, Beijing, 100080, \\ People's Republic of China
}

(Received April 15, 1996)

\begin{abstract}
A chemical modified polysaccharide hydroxylpropyl giar (HPG) derived from the guar which is a galactomannan polysaccharide obtained from seed endosperm was used to investigate the chemical cross-linked gelation. The backbone of HPG consists of 1,4 linked $\beta$-D-mannopyranosyl $\left({ }^{4} \mathrm{C}_{1}\right.$ chair conformation) residues to form a polymer chain with irregular and incomplete pendant 1,6 linked $\alpha$-D-galactopyranosyl $\left({ }^{4} \mathrm{C}_{1}\right.$ chair conformation) side groups. A viscosity enhancement was found a borate-cross-linked HPG gels which have experienced a shear flow at the early stage of chemical cross-linking. The enhancement of viscosity seems to relate with a conformational change from a spherical shape to an elongated rod-like or/and an ellipsoidal shape at the early stage of chemical cross-linking processes.

KEY WORDS Polysaccharide / Hydroxylpropyl Guar / Shear Flow / Chemical Cross-Linked Gelation /

Viscosity /
\end{abstract}

Hydroxylpropyl guar (HPG) is a family of a chemically modified polysaccharide derived from a guar. Guar is a galactomannan polysaccharide isolated from seed endosperm. ${ }^{1}$ The backbone consists of 1,4 linked $\beta$ D-mannopyranosyl $\left({ }^{4} \mathrm{C}_{1}\right.$ chair conformation) residues to form polymer chains with irregular and incomplete pendant 1,6 linked $\alpha$-D-galactopyranosyl $\left({ }^{4} \mathrm{C}_{1}\right.$ chair conformation) side groups. The structural unit of galactomannan is shown in Figure 1. The ratio of galactose to mannose has been reported to be in the region of $2: 3 .^{2}$ Mannose-rich regions have a tendency to crystallize and form insoluble residues in solution, ${ }^{3}$ because of a hydrogen bond between mannose chains.

The hydroxypropyl groups strictly prevent crystallization and result in reduced insoluble residues in solution. Figure 1 shows hydroxyl groups that react with propylene oxide under basic conditions (etherification) to form HPG. The replacement of the propylene oxide groups on guar was investigated by Albershein. ${ }^{4}$ Significant changes in properties, e.g., gelation, occur when the guar gum molecule is substituted with hydroxypropyl groups.

HPG is gelled by metal ions and is widely used in the petroleum industry, ${ }^{5}$ to transport solid particles into fractures in subterranean oil formations in a process, so called hydraulic fracturing. The important data required for the field application of fracturing fluid gels is viscosity because these results are used in numerical simulations to determine geometry, pumping rates, and proppantsetting characteristics. The effect of shear history on cross-linking has been considered substantially. ${ }^{6}$

Borate ions have long been known to form complexes with cis-diol groups (hydroxyl pairs) and with polyhydroxyl compounds in solution. ${ }^{7}$ Other types of chemical cross-linked complexes were reported by Pezron et $a l .{ }^{8,9}$ The rheological properties of the borate-crosslinked gels depend strongly on the number of effective cross-links, i.e., the concentration of $\mathrm{A}_{2} \mathrm{~B}-$, where $\mathrm{A}$ refers to the cis-hydroxyl(polyol) and $\mathrm{B}^{-}$to $\mathrm{B}(\mathrm{OH})^{-}$.

The exhibit fluid-like behavior at low $\mathrm{pH}$ (about $\mathrm{pH}$ 7.0) and solid-like behavior at high $\mathrm{pH}$ (about $\mathrm{pH}$ $9.0-9.5$ ), and a similar transition from fluid-like to solid-like behavior is seen as the temperature is decreased from $65^{\circ} \mathrm{C}$ to $15^{\circ} \mathrm{C}$ (see Kesavan et al. $1992^{10}$ ). This work presents a study of the rheological properties of boratecross-linked HPG gels which have experienced shear flow at the early stages of the chemical cross-linking process. This approach (which considered shear flow on molecular conformation before chemical cross-linking is completed) is different from Prud'omme's. ${ }^{6}$

\section{Materials}

An aqueous solutions $(0.4 \mathrm{wt} \%)$ were prepared from HPG (obtained from Dowell Schlumberger Inc.), without further purification. The weight-average molecular weights estimated by light scattering were found to be around $2.2 \times 10^{6}$ and the sample was known to be poly-disperse with $M_{w} / M_{n}$ estimated at $1.9 .{ }^{9}$ Average radius of gyration was also estimated to be around 190 nm. ${ }^{9}$ Borate solutions were prepared using boric acid. All of the present results were obtained for polymer solutions which were prepared in distilled water containing $0.18 \mathrm{wt} \% \mathrm{NaOH}$. $\mathrm{NaOH}$ was subsequently used for adjusting for $\mathrm{pH}$ of the solution.

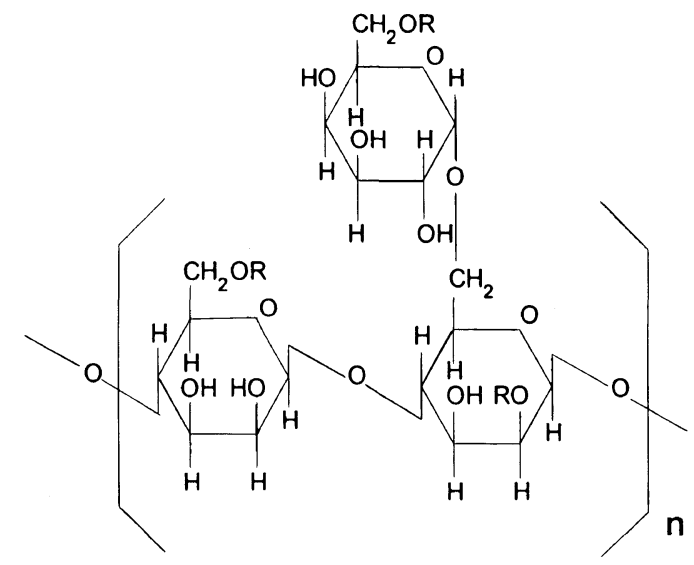

Figure 1. Trisaccharide monomer of HPG. Where $R$ is $\left(\mathrm{CH}_{2}\right)_{3} \mathrm{OH}$. 


\section{RHEOLOGICAL MEASUREMENTS}

\section{Rheometer}

The rheological behavior of the polymer solutions and the gels was characterized by determining the dynamic module in the frequency range $10^{-3} \mathrm{~Hz}$ to $10 \mathrm{~Hz}$. A shorter frequency range avoids sample degradation with time and above $10 \mathrm{~Hz}$ the control of the inertia correction is delicate at room temperature. Measurements were performed at controlled amplitude deformation (strain $<30 \mathrm{rad}$ ) and were carried out on a Rheometrics Inc. Rheometer (RMS-800) using a cone and plate geometry, with a pan radius of $25 \mathrm{~mm}$. The solution is loaded into the rheometer, and sufficient time (about $10 \mathrm{~min}$ ) was allowed for the stress in order to relax to obtain thermal equilibrium. Strain sweep experiments have also been performed before each dynamic test in order to select strain amplitudes for measurements.

\section{Mixing Procedure}

Homogeneous mixing is crucial in the formation of HPG gels. If the mixing time is longer than the reaction time, an in-homogeneous gel structure is produced. Electron microscopic studies showed non-uniform lamella and striations that were produced by inadequate mixing (see Proh'omme et al. 19896). Zasadzinski et al. ${ }^{11}$ studied gel network morphology after shear flow. They used a microliter syringe $(1 \mathrm{ml})$ to mix chemical crosslinker and polymer solutions to produce reproducible gels. These experiments were focused on the relationship between shear and rheological properties of chemical cross-linked gel. The work reported here is a preliminary study of the rheological properties taken under the shear flow at the early stage of borate-HPG gelation.

\section{RESULTS AND DISCUSSIONS}

Figure 2 is plotted of viscosity $v s$. time data and shows the difference between two different gelation procedures whether the borate HPG solution experienced a shear flow, $150 \mathrm{~s}^{-1}$ for $9 \mathrm{~min}$, at the early stage of chemical cross-linking. Firstly, without any shear history on preparing the borate-HPG gel, the gelation procedure shows the liquid state (HPG sol, $0.4 \mathrm{wt} \%$; marked $\mathrm{A}$ in Figure 2), chemical cross-linking period (marked C) and gel state (marked D). At the point of injection boric acid

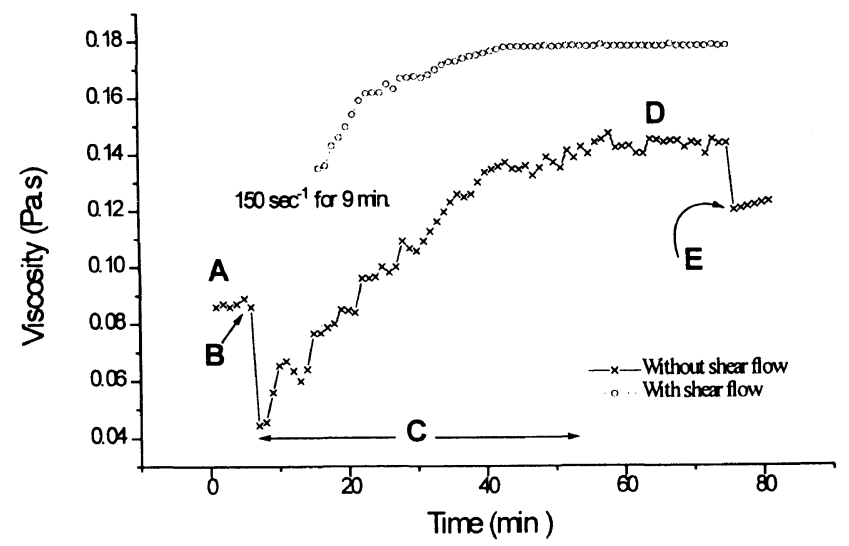

Figure 2. Gelation processes of borate-HPG (viscosity vs. time): HPG $0.4 \mathrm{wt} \%$, boric acid $0.25 \mathrm{wt} \%$ and $\mathrm{NaOH} 0.18 \mathrm{wt} \%$. solution $(0.25 \mathrm{wt} \%$ boric acid and $0.18 \mathrm{wt} \% \mathrm{NaOH}$ solution) of the viscosity drops (lower than liquid state; marked B) caused by the drop of the HPG concentration before chemical cross-linking. The viscosity of the liquid state is estimated $8.6 \times 10^{-2} \mathrm{~Pa} \cdot \mathrm{s}^{-1}$. The viscosity of the gel state is $14.3 \times 10^{-2} \mathrm{~Pa} \cdot \mathrm{s}^{-1}$. A reduction in the viscosity of chemical cross-linked gel was observed when applied shear flow after completing gelation (marked E). The phenomena has previously been observed under shear condition. ${ }^{6}$ An applied shear of $30 \mathrm{~s}^{-1}$ for two min. Produced a viscosity reduction of $3 \times 10^{-2} \mathrm{~Pa} \cdot \mathrm{s}^{-1}$, which results from the degradation of the gel formed during the shear flow.

When a shear flow $\left(150 \mathrm{~s}^{-1}\right.$ for $\left.9 \mathrm{~min}\right)$ is applied to a HPG solution $(0.4 \mathrm{wt} \%)$ at the point of injecting boric acid $(0.25 \mathrm{wt} \%)$ and $\mathrm{NaOH}(0.18 \mathrm{wt} \%)$ solution, the viscosity was increases to $17.8 \times 10^{-2} \mathrm{~Pa} \cdot \mathrm{s}^{-1}$, which is 1.25 times greater than that of the liquid state. A similar behavior was found on comparison between fast- and delayed-cross-linked gels. ${ }^{6}$ The viscosity of delayedcross-linked gel was enhanced by 2.5 times than that of the fast-cross-linked gel which also increases the viscosity rather than the fast-cross-linked gel. Therefore the enhancement of viscosity in our work would be caused by delayed-cross-linking.

A viscosity of shear treated borate-HPG gel was examined at different shear rates $(50,100,150,200,250$, and $300 \mathrm{~s}^{-1}$, respectively) and different applied times (1, 3,6 , and $9 \mathrm{~min}$, respectively) as shown in Figure 3 . The resuits show a viscosity enhancement of gels as an applied shear and/or time increase. A gelation process has a sigmoidal transition in terms of applied time and applied shear rate. A sigmoidal shaped data was fitted by the sigmoidal equation. The mid-point of a gel enhancement as a function of applied time and applied shear displays in Table I.

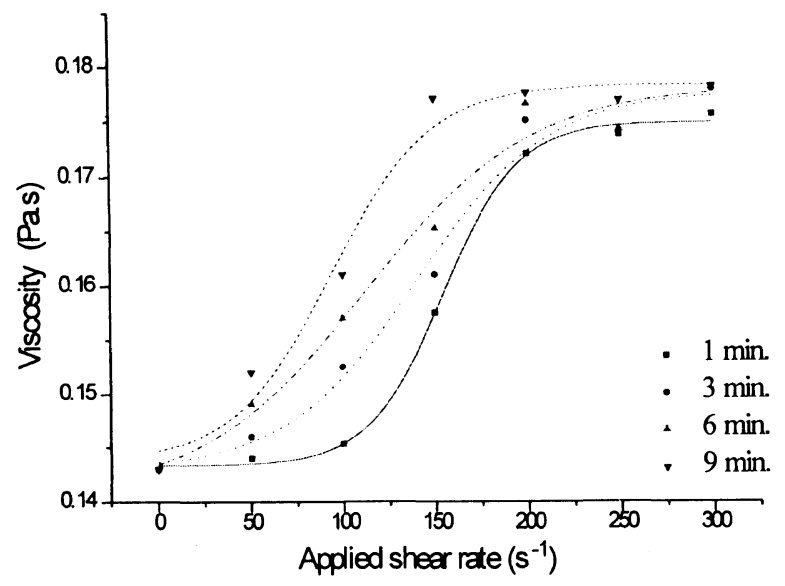

Figure 3. Viscosity enhancement of borate-HPG gel by applied shear with different applied time.

Table I. The mid-point of gel enhancement in different applied shear and time at the early stage of gelation procedure

\begin{tabular}{cc}
\hline Applied time $/$ min & Applied shear $/ \mathbf{s}^{-1}$ \\
\hline 1 & 151.3 \\
3 & 136.9 \\
6 & 103.9 \\
9 & 87.8 \\
\hline
\end{tabular}




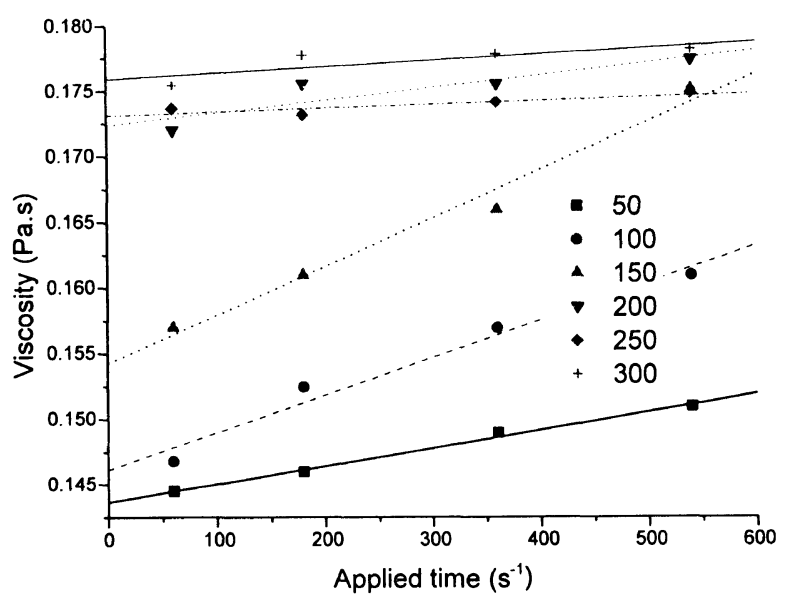

Figure 4. Applied time effects on gel enhancement.

Figure 4 shows a plot of viscosity against applied time of different shear rate. Above $200 \mathrm{~s}^{-1}$ (applied shear rate), these results do not depend strongly on applied time. Below $200 \mathrm{~s}^{-1}$, the enhancement of viscosity shows a stronger dependency upon applied time. By comparison with Figure 3, we could see that the viscosity enhancement depends more on applied shear rate than applied time.

The overlap concentration $\left(C^{*}\right)$ for HPG was measured by Pezron et al. ${ }^{9}$ which was estimated at $0.11 \mathrm{wt} \%$ (cf. $0.07 \mathrm{wt} \%$ by Zasadzinski et al. ${ }^{11}$ ), for the same $M_{w}$ used in this study $\left(2.2 \times 10^{6}\right)$. In this work, $0.4 \mathrm{wt} \% \mathrm{HPG}$ solution was chosen. Above overlap concentration, in order to reach the gelation threshold roughly two intermolecular cross-linkes are needed per HPG chain. The number density of intermolecular cross-links $\left(N_{\text {inter }}\right)$, we assume, is proportional to the gel viscosity. $N_{\text {inter }}$ can be explained as a function of free borate concentration $\left(\left[\mathrm{B}^{-}\right]\right)$, HPG concentration $(C)$ and temperature $(T)$. Pezron et al. ${ }^{9}$ derived the relation:

$$
N_{\text {inter }} \sim\left[\mathrm{B}^{-}\right] C^{3 \mu /(3 \mu-1)} \exp (-\Delta H / R T)
$$

where $\Delta H$ is the enthalpy of formation of a cross-linked and $\mu$ is the swelling exponent relating the radius of gyration $\left(R_{\mathrm{g}}\right)$ and the molecular weight of the chains. In this work, $\left[\mathrm{B}^{-}\right], C$, and $T$ are constant. The value of $\mu$ will be changed with applied shear at the early stage of cross-linking. If we only consider the variation of the value of $\mu, N_{\text {inter }}$ can be written as

$$
N_{\text {inter }} \sim C^{3 \mu /(3 \mu-1)}
$$

Using the value $\mu$ obtained from above relations and Figure 3 (for $6 \mathrm{~min}$ ), $\left(R_{\mathrm{g}} / R\right)^{2}$ was calculated based on the equation, ${ }^{12}$ for $r^{*}<r<1$,

$$
\left(R_{\mathrm{g}} / R\right)^{2}=1 / 6+\left(C / C^{*}\right)^{3 /(1-d \mu)}\{1 /(2 \mu+1)(2 \mu+2)-1 / 6\}
$$

where $d$ is the space dimension $(d \leq 4), R_{\mathrm{g}}$ is the radius of gyration which is defined as the square root of the mean square distance of all individual elements of the HPG chain from the center of gravity of each HPG chain and $R$ is the end-to-end distance (or displacement length) of chain. The space dimension $d=2$ was plotted in Figure 5. All data of $\left(R_{\mathrm{g}} / R\right)^{2}$ are related not to the gel shape but to the HPG shape at the early stage of chemical cross-linking process. The reduction of $\left(R_{\mathrm{g}} / R\right)^{2}$ means that the increment of $R$ is greater than that of $R_{\mathrm{g}}$ as the

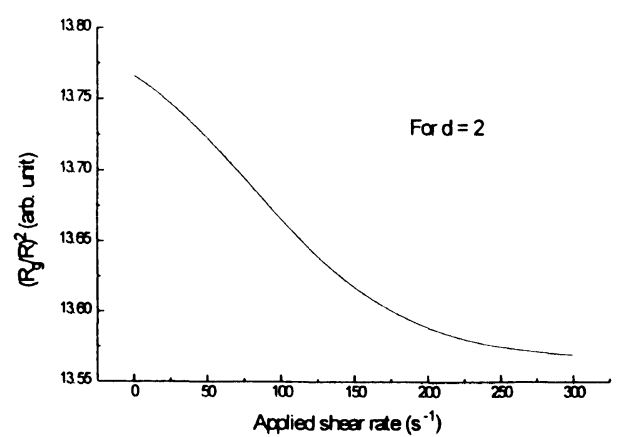

Figure 5. Plot of $\left(R_{\mathrm{g}} / R\right)^{2} v s$. applied shear rate with space dimension, $d=2$.

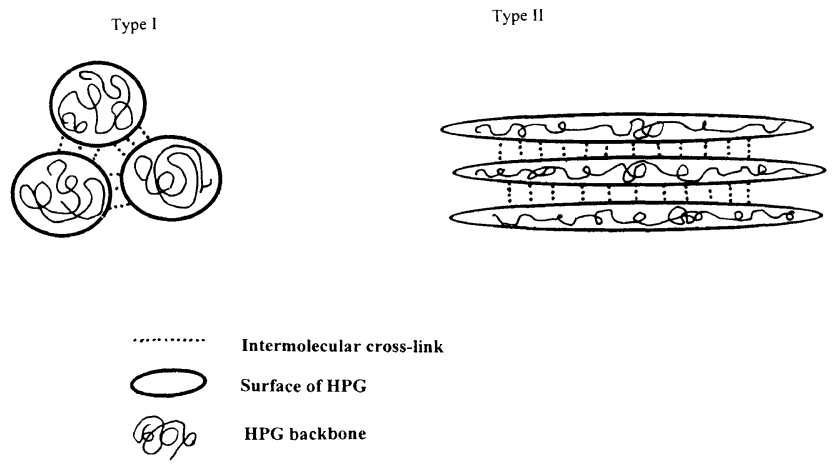

Figure 6. Possible gelation mechanisms of the chemical cross-linked HPG. Type I has not experienced a shear flow at the early stage of chemical cross-linking procedure, but type II has.

applied shear rate is increased. Consequently, the conformational transform of HPG molecules may be corresponding to the change from a sphere-shaped chain to an elongated rod-like (and/or an ellipsoid-shaped) chain. Therefore, the enhancement of viscosity seems to relate with the increment of the surface of each individual HPG molecule at the early stage of chemical cross-linking process. Consequently, the increment of the surface of each individual HPG molecule produce higher density of chemical cross-linkage, $N_{\text {inter }}$, with neighboring molecules. As a shear rate increases, that $R$ increases faster than $R_{\mathrm{g}}$, which is related with the chain elongation. Two types of the chemical cross-linked gelation were illustrated in Figure 6. The gelation type I may obtained from the sample which has not experienced the shear flow at the early stage of gelation procedure. The elongated getation, type II, may be produced by the shear flow before forming the chemical cross-links. It could be that $N_{\text {inter }}$ of the type II is higher than that of the type I because of the increment of the intermolecular contact. Based on above assumptions, attempts were made during shear flow of HPG solution to detect any birefringence caused by the change of chain conformation. However, the birefringence caused by the conformational changes were relatively low intensity that was not enough to identify. Even when highly concentrated HPG solution $(1-2 \mathrm{wt} \%)$ were used, the intensity of birefringence was less than that of scattering by shear cell window (using optical window).

Acknowledgment. This work has been carried out with the financial support from Chinese Academy of Sciences and Natural Science Foundation of China. One 
of us (IL) is grateful for the support from State Bureau of Foreign Experts, People's Republic of China.

\section{REFERENCES}

1. A. Goldstein and W. Stivers, "Guar Gum in Petroleum Production," Celanese Co., Jeffersontown, Kenterky, 1980.

2. T. J. Paints, Lebensm-Wiss Technol., 15, 57 (1982).

3. E. R. Morris, Br. Polym. J., 18, No. 1, 14 (1986).

4. A. Albershein, Carbohyd. Res., 131, 139 (1984).

5. J. K. Borchardt, Am. Chem. Soc., Sym. Ser., 196, 3 (1989).

6. R. K. Prud'omme, V. Constein, and S. Knoll, ACS Sym. Ser.,
223, 89 (1989)

7. H. Deuel and H. Neukem, Makromol. Chem., 3, 113 (1949).

8. E. Pezron, A. Richard, F. Lafund, and R. Audebert, Macromolecules, 21, 1121 (1988a).

9. E. Pezron, F. Lafund, A. Richard, and R. Audebert, Macromoleules, 21, 1126 (1988b).

10. S. Kesavan and R. K. Prud'omme, Macromolecules, 25, 2026 (1992).

11. J. A. Zasadzinski, A. Chu, and R. K. Prud'omme, Macromolecules, 19, 2960 (1986).

12. M. Daoud, J. P. Cotton, B. Farnoux, G. Jannink, G. Sarma, R. Benoit, R. Duplessix, G. Picot, and P. G. de Gennes, Macromolecules, 8, 804 (1975). 